This is an electronic reprint of the original article. This reprint may differ from the original in pagination and typographic detail.

\author{
Author(s): Karppinen, Pasi; Salmi, Ari; Moilanen, Petro; Zhao, Zuomin; Myllylä, R.; Timonen, Jussi; \\ Hæggström, E.
}

Title: $\quad$ Phase-delayed laser diode array allows ultrasonic guided wave mode selection and tuning

Year: $\quad 2013$

Version:

Please cite the original version:

Karppinen, P., Salmi, A., Moilanen, P., Zhao, Z., Myllylä, R., Timonen, J., \& Hæggström, E. (2013). Phase-delayed laser diode array allows ultrasonic guided wave mode selection and tuning. Journal of Applied Physics, 113(14), Article 144904. https://doi.org/10.1063/1.4801394

All material supplied via JYX is protected by copyright and other intellectual property rights, and duplication or sale of all or part of any of the repository collections is not permitted, except that material may be duplicated by you for your research use or educational purposes in electronic or print form. You must obtain permission for any other use. Electronic or print copies may not be offered, whether for sale or otherwise to anyone who is not an authorised user. 


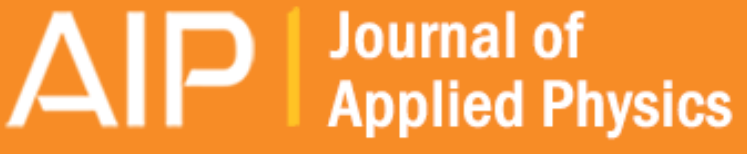

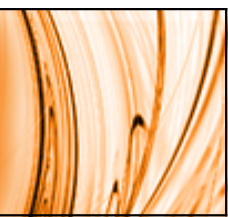

\section{Phase-delayed laser diode array allows ultrasonic guided wave mode selection and}

\section{tuning}

Pasi Karppinen, Ari Salmi, Petro Moilanen, Timo Karppinen, Zuomin Zhao, Risto Myllylä, Jussi Timonen, and

Edward Hæggström

Citation: Journal of Applied Physics 113, 144904 (2013); doi: 10.1063/1.4801394

View online: http://dx.doi.org/10.1063/1.4801394

View Table of Contents: http://scitation.aip.org/content/aip/journal/jap/113/14?ver=pdfcov

Published by the AIP Publishing

\section{Articles you may be interested in}

Amplitude controlled array transducers for mode selection and beam steering of guided waves in plates

AIP Conf. Proc. 1511, 215 (2013); 10.1063/1.4789051

Guided wave mode pairs for transmissibility in adhesively bonded metal plates

AIP Conf. Proc. 1511, 199 (2013); 10.1063/1.4789049

Two-dimensional broadband distributed-feedback quantum cascade laser arrays

Appl. Phys. Lett. 98, 141101 (2011); 10.1063/1.3574555

Spatially resolved and temperature dependent thermal tuning rates of high-power diode laser arrays Appl. Phys. Lett. 88, 133510 (2006); 10.1063/1.2190454

Stability of the single-mode output of a laser diode array with phase conjugate feedback

Appl. Phys. Lett. 76, 535 (2000); 10.1063/1.125810

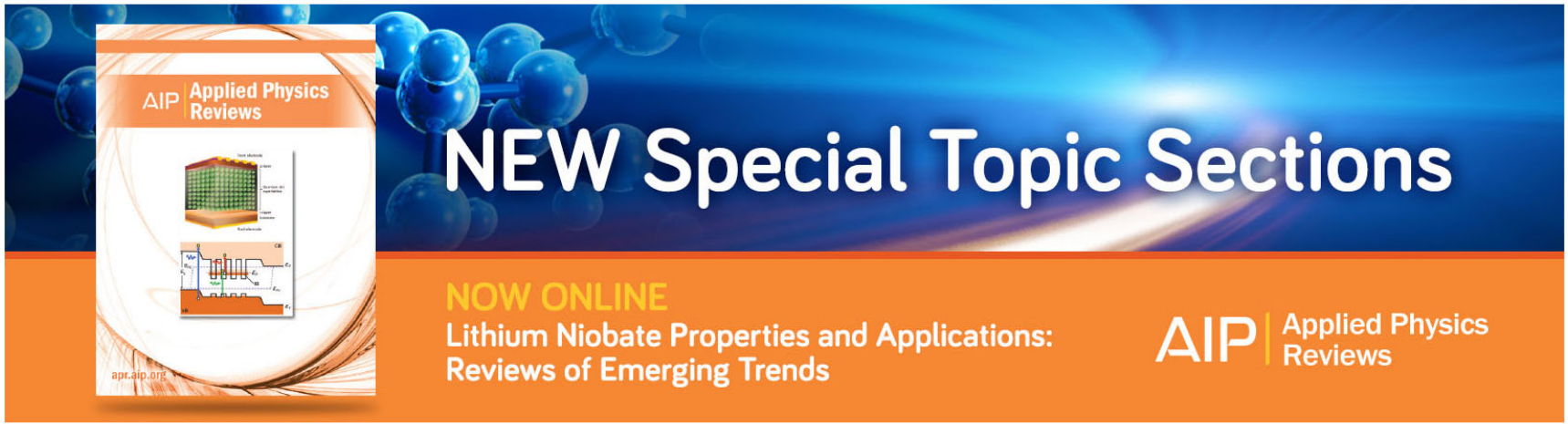




\title{
Phase-delayed laser diode array allows ultrasonic guided wave mode selection and tuning
}

\author{
Pasi Karppinen, ${ }^{1, a), b)}$ Ari Salmi, ${ }^{1, a)}$ Petro Moilanen, ${ }^{2}$ Timo Karppinen, ${ }^{1}$ Zuomin Zhao, ${ }^{3}$ \\ Risto Myllylä, ${ }^{3}$ Jussi Timonen, ${ }^{2}$ and Edward Hæggström ${ }^{1}$ \\ ${ }^{1}$ Electronics Research Laboratory, Department of Physics, Division of Materials Physics, \\ University of Helsinki, P.O.B. 64, Uusimaa FIN-00014, Finland \\ ${ }^{2}$ University of Jyväskylä, P.O.B. 35, 40014 Jyväskylä, Finland \\ ${ }^{3}$ Department of Electrical Engineering, University of Oulu, Oulu, Finland
}

(Received 7 February 2013; accepted 26 March 2013; published online 11 April 2013)

\begin{abstract}
Selecting and tuning modes are useful in ultrasonic guided wave non-destructive testing (NDT) since certain modes at various center frequencies are sensitive to specific types of defects. Ideally one should be able to select both the mode and the center frequency of the launched waves. We demonstrated that an affordable laser diode array can selectively launch either the $\mathrm{S}_{0}$ or $\mathrm{A}_{0}$ ultrasonic wave mode at a chosen center frequency into a polymer plate. A fiber-coupled diode array (4 elements) illuminated a $2 \mathrm{~mm}$ thick acrylic plate. A predetermined time delay matching the selected mode and frequency was employed between the output of the elements. The generated ultrasound was detected by a $215 \mathrm{kHz}$ piezo receiver. Our results imply that this array permits non-contacting guided wave ultrasonic NDT. The solution is small, affordable, and robust in comparison to conventional pulsed lasers. In addition, it does not require experienced operators. (C) 2013 AIP Publishing LLC. [http://dx.doi.org/10.1063/1.4801394]
\end{abstract}

\section{INTRODUCTION}

Guided waves $(\mathrm{GW})$ are employed for non-destructive testing (NDT) and structural health monitoring (SHM) as they provide information about structural and elastic properties of the examined bodies. Since GWs can probe much of a guiding structure from a single or a few insertion points, and since GWs are sensitive to small discontinuities in the mechanical impedance along the propagation path, they can rapidly inspect even large constructs. For example, GWs can detect cracks or corrosion damage in a long pipe or an aircraft wing with a single measurement. ${ }^{1}$ Since GWs are influenced by the mechanical boundary conditions of the guiding structure, they can sense modifications at its interfaces or surfaces. This capacity has been used, e.g., to determine the quality of adhesive bonds ${ }^{2}$ and to inspect composite materials such as aircraft parts. ${ }^{3}$

Ultrasonic NDT benefits from rapid methods that are sensitive and that provide access to hard-to-reach sites. This goal can be achieved, e.g., by optically exciting guided sound waves (GWs), which enables remote, non-contacting, and galvanically isolated measurements. ${ }^{4}$ Maximum sensitivity to detect a certain flaw is achieved by launching a specific ultrasonic mode. ${ }^{5}$ To this end one needs to control the particle displacement in the sample (direction and amplitude) at precisely determined sites. This ability to selectively generate modes with a specific frequency band and spatial pattern-called mode tuning - is important for quantitative GW inspection. ${ }^{6}$ Once tuned, one can apply a specific mode featuring a preselected center frequency, which provides maximum sensitivity for revealing and assessing a certain structural condition

\footnotetext{
${ }^{\text {a) }}$ P. Karppinen and A. Salmi contributed equally to this work.

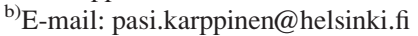

(flaw, damage, crack, disbond). Such mode tuning can be accomplished either by the angle beam method (contacting transducers $)^{7}$ or by spatially and/or temporally modulating the excitation signal (either contacting or non-contacting transducers). ${ }^{8}$ This approach can in principle be used with both linear and nonlinear ultrasound measurement techniques.

Contact guided wave NDE techniques typically employ piezo-ceramic or polyvinylidene-fluoride (PVDF) transducers. There are three common techniques for mode tuning with contact transducers:

(1) One selects the incidence angle of the transmitted sound (angle beam method) to excite the desired mode into the sample. $^{7}$ The downside of this method is that several wedges or an adjustable wedge are needed if one wants to launch different modes.

(2) Mode tuning by spatial modulation of the excitation can be done with interdigital transducers (IDTs) ${ }^{9,10}$ or by comb transducers. ${ }^{11}$ However, these transmitters must be physically reconfigured or exchanged every time a different mode is to be excited.

(3) Time-delay excitation combines spatial and temporal modulation, ${ }^{8,12-14}$ in which mode tuning is achieved with an electronic time delay circuit. This approach allows rapid reprogramming of the time delays to reconfigure the transmitter for launching a certain mode.

Contact methods often face challenges with, e.g., fragile, hot or moving samples or with samples featuring poorly defined or rough surfaces. These challenges may be solved by measurements done without contact; non-contact mode tuned ultrasonic guided wave measurements can be performed using, e.g., electro-magneto-acoustic transducers (EMATs) ${ }^{15}$ airborne ultrasound transducers, ${ }^{16}$ and lasers. ${ }^{17}$ In addition, flashlamp-based excitation ${ }^{18}$ could be used for 
mode tuning with suitable masks. EMATs permit spatial modulation of the transmitted signal whereas in airborne ultrasound measurements one can select the angle of sound incidence to have mode tuning. In contrast to EMATs and airborne ultrasound transmitters, lasers excite a broadband ultrasonic signal that can be concentrated into a narrow frequency band (by using a mask to generate several line sources). ${ }^{19}$ Laser excitation also allows a larger stand-off than the previous modalities. ${ }^{20}$

To achieve laser ultrasonic mode tuning, spatial modulation of the laser excitation can be done with lenticular lenses, ${ }^{17,21}$ optical diffraction gratings, ${ }^{22}$ laser interference patterns, ${ }^{23}$ and transmission masks. ${ }^{19}$ Temporal laser beam modulation can be done with a Bragg cell ${ }^{24}$ or by modulating the intensity of a continuous wave $(\mathrm{CW})$ laser. ${ }^{25}$ Combined spatial and temporal modulation has been achieved using fiber optical delay lines ${ }^{26}$ multiple lasers, ${ }^{27}$ or an acoustooptical modulator. ${ }^{28}$

Of the non-contact methods, EMATs can excite sound only into electrically conducting materials, from a close and precisely controlled distance off the sample. Airborne ultrasound has signal-to-noise-ratio (SNR) issues due to the large difference in acoustic impedance between air and most inspected materials as well as due to the attenuation of high frequency ultrasound in air. On the other hand, laser ultrasonic excitation offers a valuable tool for NDT and SHM, especially when combined with a non-contacting detection method like an interferometer or a Doppler vibrometer. Pulsed lasers are unfortunately expensive, which generates a need for an inexpensive, mode tunable, laser GW excitation technique. Mode tuning also addresses the SNR issue often present with these noncontact methods.

Solid state light sources find many applications, which explains the rapid progress in the field of solid state light source engineering. ${ }^{29}$ In NDT, solid state light sources, particularly laser diodes, have already found use. ${ }^{30,31}$ These sources, at least partially, could address shortcomings of Nd:YAGs employed for guided wave NDT. They are cheap, small, rugged, and allow fast modulation of intensity and wavelength. Laser diodes have been proposed as a cheap and compact alternative to somewhat expensive and bulky Q-switched Nd:YAGs for laser ultrasonic excitation. ${ }^{31}$ The main disadvantage of using laser diodes for such excitation is their low peak power compared to that of Q-switched lasers. The SNR in a laser diode based GW measurement can be improved by using coded signals to modulate the $\mathrm{CW}$ laser diode. ${ }^{25}$

To address the previously mentioned needs we set out to demonstrate that an array of four pulsed laser diodes can selectively excite guided waves (Ao and So plate modes) into an acrylic plate featuring a predetermined center frequency. This work strives to introduce an inexpensive laser acoustic GW excitation method that permits fast and flexible mode tuning. These features should be interesting in noncontact ultrasonic material testing and characterization.

\section{METHODS}

Our sample was a $2.0 \pm 0.1 \mathrm{~mm}$ thick black acrylic plate (Plexiglas type 9N870, Etra, Inc.) fixed onto a steel frame that supported the plate at its edges. The acoustic impedance of the plate was 3.2 MRayl, whereas the optical absorption coefficient was sufficient to absorb $905 \mathrm{~nm}$ laser light in a thin layer near the surface of the sample. The excitation head consisted of four pulsed $905 \mathrm{~nm}$ laser diodes (SPL PL 90_3, OSRAM Opto Semiconductors, $7 \mathrm{~nm}$ spectral width) that were driven with four laser drivers (PCO - 7120, Ixys Colorado). Laser light was introduced into a $200 \mu \mathrm{m}$ core diameter multimode fiber (M27L02, Thorlabs) whose distal end was mounted into a linear fiber array $(10 \mathrm{~mm}$ inter-fiber spacing), see Fig. 1. The laser diodes emitted $500 \mathrm{~ns}$ long pulses every millisecond $(3.0 \pm 0.1 \mathrm{~A}$ drive current). The $(10 \%-90 \%)$ rise time of the laser diode was $1 \mathrm{~ns}$, whereas the pulse rise time of the laser diode driver was $8 \mathrm{~ns} .{ }^{32}$ The diode drivers were individually triggered with a commercial I/O module (NI 5732, National Instruments). This allowed us to control the time delay between the laser array elements with $100 \mathrm{~ns}$ resolution.
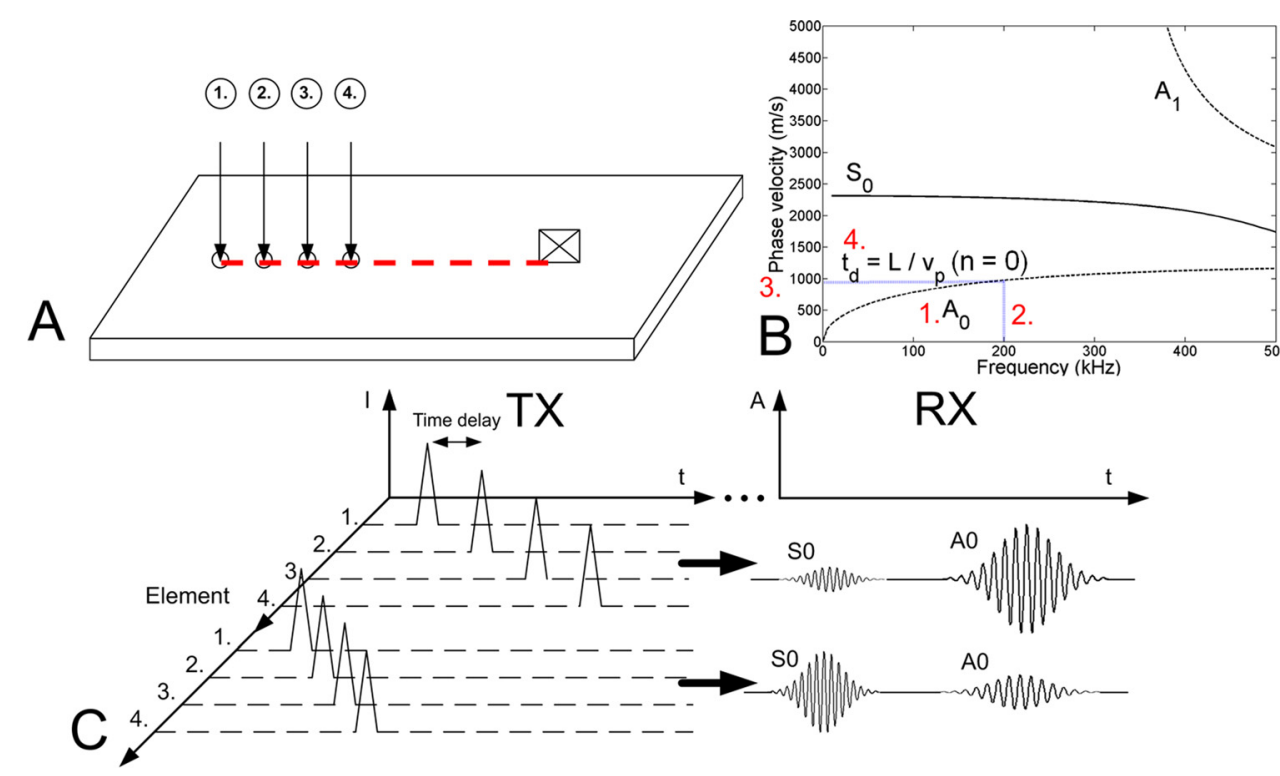

FIG. 1. (a) Measurement setup and sample geometry. The four excitation points (marked one through four) and the receiving transducer are shown. (b) Protocol for selecting the appropriate time delay. Theoretical dispersion graph for a $2 \mathrm{~mm}$ thick acrylic plate. First, the desired mode (1) and frequency (2) are selected. Then, the phase velocity matching these (3) is determined, and the time delay value is calculated (4). (C) Schematic excitation protocol. Changing the delay between the laser pulses (left) allows exciting the $S_{0}$ and $A_{0}$ modes with selectable $S_{0} / A_{0}$ power fraction, which allows mode tuning. 
Signal excitation was done using a time-delay excitation method, in which the laser diodes emitted light in a rapid succession at chosen time-delays (see Fig. 1). When the time delay (for a certain diode array) matched the phase velocity of the desired mode at a desired frequency, that mode was amplified. ${ }^{8}$ These modes were excited for any time delay $t_{d},{ }^{8}$

$$
t_{d}=\frac{L}{v_{p}}-n \frac{1}{f},
$$

where $\mathrm{L}$ is the inter-element distance, $\mathrm{f}$ is the selected frequency, $\mathrm{v}_{\mathrm{p}}$ is the phase velocity of the selected mode at that frequency, and $\mathrm{n}$ is an integer. This is depicted in Fig. 2.

The fiber array faced the acrylic plate at right angle. The distance between the plate and each collimating lens at the fiber tips was $2 \mathrm{~mm}$, resulting in four light spots less than $1 \mathrm{~mm}$ by diameter. Optically generated ultrasonic waves were picked up with a contacting piezo transducer (custom made transducer, $215 \mathrm{kHz}$ center frequency, $180 \mathrm{kHz}-240$ $\mathrm{kHz}-6 \mathrm{~dB}$ bandwidth, $10 \mathrm{~mm}$ face plate diameter). The piezo transducer was aligned with the fiber array, i.e., its center was placed on a straight line traversing the centers of all fibers and the receiving transducer. The distance between the element of the fiber array that was closest to the receiver and the center of the piezo transducer element was $20.0 \pm 0.5 \mathrm{~mm}$. Ultrasonic gel (Aquasonic 100, Parker Laboratories, Inc.) enhanced the acoustic coupling between the receiver and the acrylic plate. To obtain a 2D-FFT representation of the received signals, the diode array was translated with $0.25 \mathrm{~mm}$ steps along the sample surface with a Micos VT-80 translation stage.

For each measurement point, the following protocol was performed: First, each of the four transducers emitted a laser pulse by themselves (this was done to obtain a reference value). Second, the entire diode array excited pulses with pre-selected time delay values ranging from 0 to $20 \mu \mathrm{s}$ (100 ns steps). Third, the diode array was translated to the next location (160 distances were measured in total). The entire measurement was repeated three times. The signal

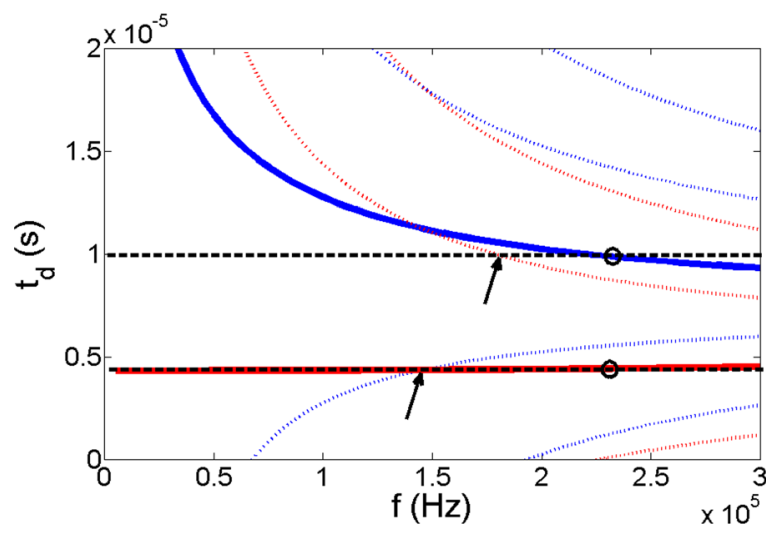

FIG. 2. The figure explains how unintended higher integer time delay excitation may take place. Blue lines indicate $\mathrm{A}_{0}$ mode and red lines the $\mathrm{S}_{0}$. The solid lines represent $n=0$, whereas the dotted ones represent $n \neq 0$ excitation (modes higher in $t_{d}$ are $n>0$ ). At a time delay, corresponding to the selected modes and frequencies $(230 \mathrm{kHz}$, black dotted lines), the unselected modes are unintentionally excited at $n=-1$ ( $S_{0}$ selected, expected frequency $145 \mathrm{kHz})$ and $\mathrm{n}=1\left(\mathrm{~A}_{0}\right.$ selected, expected frequency $\left.180 \mathrm{kHz}\right)$, marked with arrows. recorded by the contact transducer was first filtered $(0.5 \mathrm{kHz}$ high-pass analog filter) and then amplified in two stages ( $43 \mathrm{~dB}$ first stage, custom made amplifier, and $31 \mathrm{~dB}$ second stage, Panametrics 5072PR). The amplified signal was digitized with the digitizer adapter of the NI $5732(80 \mathrm{MHz}, 14$ bits) and averaged 1024 times prior to data analysis. The measured data were first down-sampled to $8 \mathrm{MHz}$ and then zero-padded (we added 1000 signals with all zeros to the 160 distances measured and 5000 zero points to the end of each measured signal). Next a 2D-FFT power spectrum (square of the magnitude of the 2D-FFT) of the 1160 by 6000 element data matrix was generated using a custom made LABVIEW (National Instruments, Inc.) - program (no windowing was used). The frequency and wavenumber resolutions of the 2D-FFT spectrum were $1.3 \mathrm{kHz}$ and $3.4 \mu \mathrm{m}^{-1}$.

At each frequency, the time delay producing the maximum power in either the $S_{0}$ or $A_{0}$ (selected mode, $n=0$ ) within a $0.34 \mathrm{~mm}^{-1}$ wide wave number window was determined. The expected locations in the (f-k) graph for these modes were theoretically determined from the Lamb wave dispersion equations, ${ }^{33}$ and the window width was chosen to exclude the non-selected mode. The obtained power value was then compared to the value of the non-selected mode generated using this particular time delay ("mode tuned power ratio"). Finally, we determined the power ratio of the $\mathrm{A}_{0}$ and $\mathrm{S}_{0}$ ( $\mathrm{A}_{0}$ divided by $\mathrm{S}_{0}$ and vice versa) modes excited by the closest laser diode alone and divided the mode tuned power ratio by these values. The result indicates the power gain achieved using time delay based mode selection, Eq. (2),

$$
\alpha(f)=\frac{\frac{P_{S M}(f)}{P_{O M}(f)}}{\frac{P_{s l_{S M}}(f)}{P_{s l_{o M}}(f)}} .
$$

Here, $P_{s l_{S M}}$ is the power in the selected mode generated with a single laser, whereas $P_{S M}$ is the corresponding value for array generation. $P_{s l_{O M}}$ is the power in the mode that was not selected for single laser generation and $P_{O M}$ for array generation.

\section{RESULTS}

2D-FFT graphs of the received signals for either $A_{0}$ selected (10.6 $\mu$ s delay) or $\mathrm{S}_{0}$ selected ( $4.9 \mu$ s delay) as well as the signals obtained from a single laser excitation are presented in Fig. 2. Examples of signals with $\mathrm{A}_{0}$ and $\mathrm{S}_{0}$ selected are presented in Fig. 3.

The ratio between $A_{0} / S_{0}$ and $S_{0} / A_{0}$ as a function of frequency is presented in Fig. 5. The average power amplification for the $\mathrm{S}_{0}$ mode was $8.0 \pm 1.6$ (corresponding to $9 \mathrm{~dB}$ ) and $5.6 \pm 0.5$ (corresponding to $7.5 \mathrm{~dB}$ ) for the $\mathrm{A}_{0}$ mode, when compared to an excitation with a single laser. It has to be noted that the array has four diodes; theoretically, multiplying the single spot result by four reduces the recorded power gain by no more than $6 \mathrm{~dB}$.

\section{DISCUSSION}

Figure 3 demonstrates both a significant increase in the excitation power gained by 4-point array excitation 

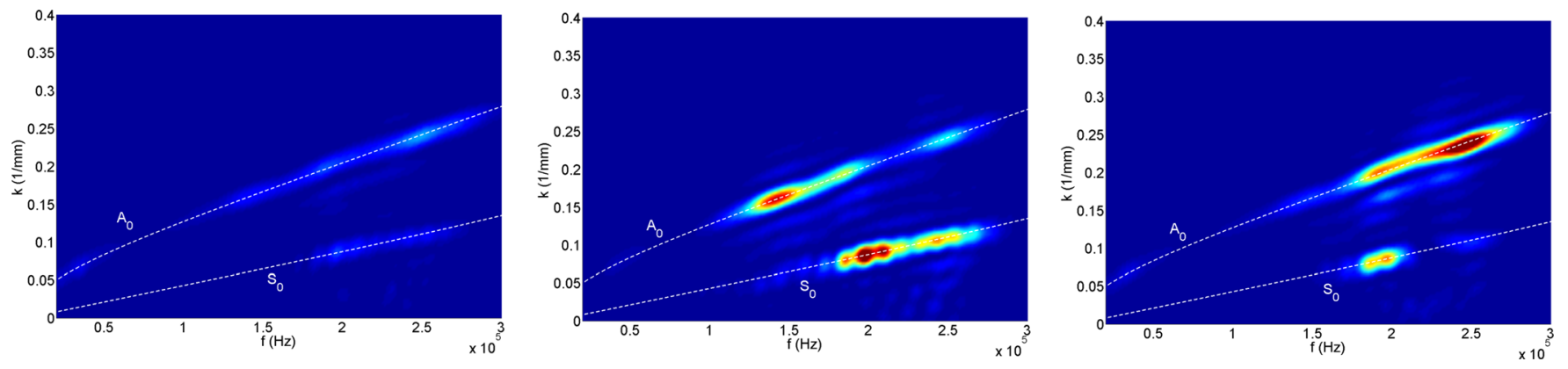

FIG. 3. Mode tuning. (left) 2D-FFT of signals generated by standard laser excitation (one laser diode as TX, no phasing). (middle) 2D-FFT of excitation done by three laser diodes with $\mathrm{S}_{0}$ as the selected mode. (right) 2D-FFT of excitation done by three laser diodes with $\mathrm{A}_{0}$ as the selected mode. The chosen center frequency was $230 \mathrm{kHz}$. The white dotted lines represent the theoretical locations for the modes (calculated from the dispersion equations). The colors are normalized to 1 (blue low-red high, linear scale).

compared to a single point excitation (compare left part to middle and to right) and the capability of mode selection. Comparing the middle and right parts of the figure shows capacity to focus power either to the $S_{0}$ mode (middle) or to the $A_{0}$ mode (right) at a freely selectable frequency (within the RX transducer bandwidth). The non-selected mode is visible outside this frequency. This is likely due to mode excitation at higher $\mathrm{n}$ values, see Fig. 2 . This capacity to select modes is also visible in Fig. 4. This figure also demonstrates the high SNR we obtained in the measurements. The effect arising from $\mathrm{n} \neq 0$ modes is visible: For $\mathrm{n}>0$, the mode arrives later (earlier for $n<0$ ) compared to $n=0$ since the furthermost laser emits first and the time delay is added to (or subtracted from when $\mathrm{n}<0$ ) the time-of-arrival of the signal.

The increase in power compared to single point laser measurements is quantified in Fig. 5. The average increase in power was smaller for the $A_{0} / S_{0}$ fraction compared to the $S_{0} /$ $\mathrm{A}_{0}$ fraction at lower frequencies $(<210 \mathrm{kHz})$. This is likely due to $n=1$ excitation of the $S_{0}$, mode since it is excited at these frequencies with the selected time delay of $A_{0}$, see Fig. 2 . Interference from modes generated by $n \neq 0$ time delays can also be reduced by proper array design (distance between excitation elements), since the inter-element distance governs the location of these modes in the time-delay space. This needs to be done for each sample class.

One important factor affecting the results is contact between the piezo transducer and the plate: since the transducer was gel coupled, it is likely that the contact varied from measurement to measurement. Such contact variation alters the power spectrum of each mode and affects which mode is dominantly detected. Nevertheless, our results are still valid since we normalized each measurement (Eq. (2)) to itself which eliminates the effects of contact variation. In addition, the finite width of the piezoelectric transducer as well as its apodized sensitivity profile along the radial direction did not bias the conclusions of this paper, since the same transducer was used in all measurements.

The method presented here is affordable and rapid: to tune a specific mode at a specific frequency, one does not have to change the positions of the array elements. In addition, pulsed laser diodes are generally Class 3B, which makes them safer than the Class $4 \mathrm{Nd}$ :YAG commonly used. We foresee applications for phased laser diode arrays e.g. in guided waves excited in bone through soft tissue ${ }^{34}$ - a problem in which modes need to be generated as purely as possible at a selected frequency. The proposed method should offer improved sensitivity (mode selection) and penetration

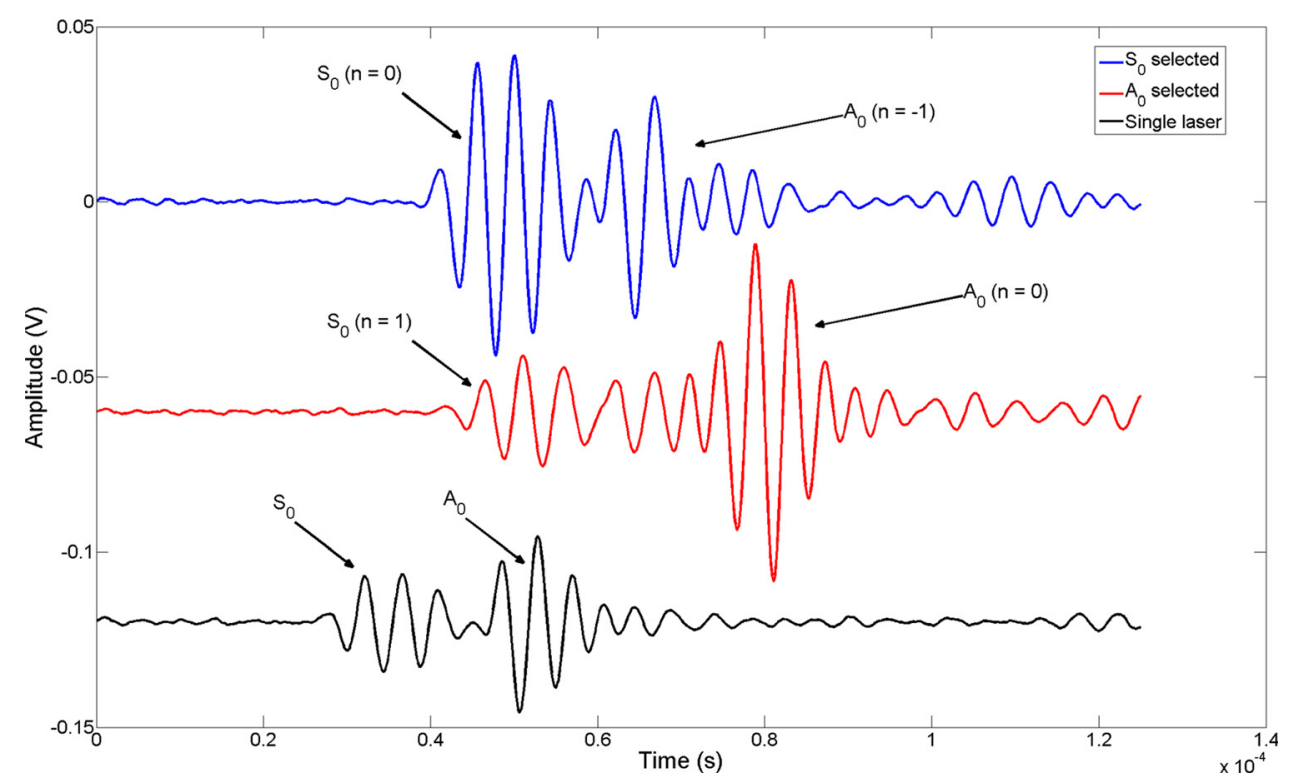

FIG. 4. Measured signals. Two different signals where either $\mathrm{A}_{0}$ or $\mathrm{S}_{0}$ mode is chosen: (top) $\mathrm{S}_{0}$ dominant and (middle) $\mathrm{A}_{0}$ dominant signal. The modes were identified using the phase velocities extracted from the 2D-FFT graph. The selected center frequency was $230 \mathrm{kHz}$. The signals are filtered with a 1 st order Butterworth digital filter $(200-260 \mathrm{kHz}$ bandpass). For comparison, a signal excited with a single laser is also shown (bottom). Signals were offset vertically by $0.06 \mathrm{~V}$ per signal for visual purposes. 


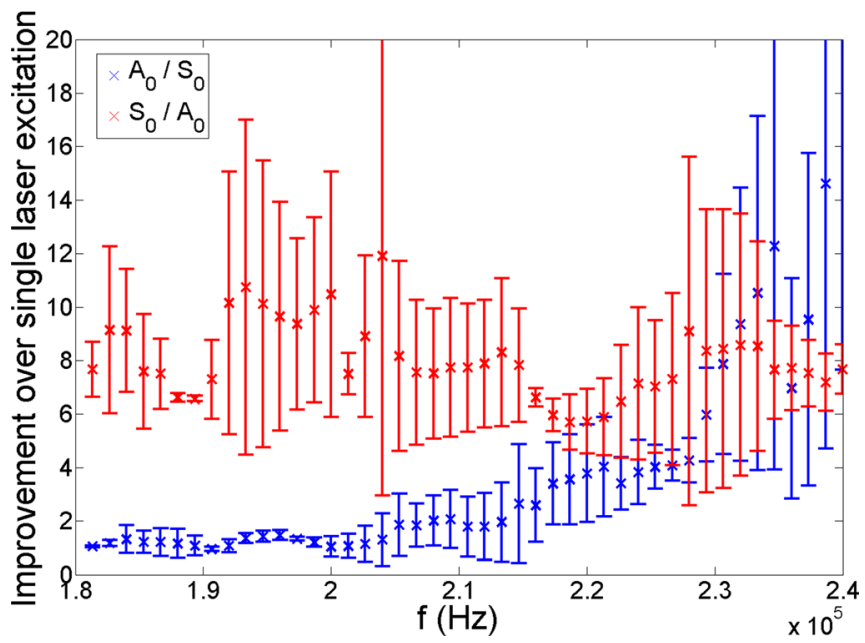

FIG. 5. Improvement over single laser excitation as a function of chosen frequency. The ratio between the powers of $\mathrm{A}_{0}$ and $\mathrm{S}_{0}$ modes normalized to the case of single laser excitation (no mode selection). The error bars represent one standard deviation from three repetitive measurements. There is an increase in the excited power ratio across the entire bandwidth of the transducer.

power (frequency), which should benefit both NDT and SHM.

\section{CONCLUSIONS}

We demonstrated a time-delayed laser diode array based method for mode tuning which is rapid and affordable. We were able to amplify the power ratio of the selected mode at a selected frequency by $10+\mathrm{dB}$ compared to single laser diode excitation.

\section{ACKNOWLEDGMENTS}

We gratefully acknowledge the financial support by the Academy of Finland via the Photonics and Modern Imaging Techniques program (project numbers 134897, 135211, and 135069).

${ }^{1}$ J. L. Rose, Trans. ASME J. Pressure Vessel Technol. 124(3), 273-282 (2002).
${ }^{2}$ T. Kundu, A. Maji, T. Ghosh, and K. Maslov, Ultrasonics 35(8), 573-580 (1998).

${ }^{3}$ Z. Su, L. Ye, and Y. Lu, J. Sound Vib. 295(3), 753-780 (2006).

${ }^{4}$ S. Davies, C. Edwards, G. Taylor, and S. B. Palmer, J. Phys. D 26(3), 329 (1993).

${ }^{5}$ T. Ghosh, T. Kundu, and P. Karpur, Ultrasonics 36(7), 791-801 (1998).

${ }^{6} \mathrm{H}$. Gao and J. Rose, Aeronaut. J. 114(1151), 49-56 (2010).

${ }^{7}$ H. J. Shin and J. L. Rose, J. Nondestruct. Eval. 17(1), 27-36 (1998).

${ }^{8} \mathrm{~J}$. Li and J. L. Rose, IEEE Trans. Ultrason. Ferroelectr. Freq. Control 48(3), 761-768 (2001).

${ }^{9}$ R. Monkhouse, P. Wilcox, and P. Cawley, Ultrasonics 35(7), 489-498 (1997).

${ }^{10}$ T. R. Hay and J. L. Rose, IEEE Trans. Ultrason. Ferroelectr. Freq. Control 53(6), 1212-1217 (2006).

${ }^{11}$ J. Rose, S. Pelts, and M. Quarry, Ultrasonics 36(1), 163-169 (1998).

${ }^{12}$ W. Luo and J. L. Rose, J. Acoust. Soc. Am. 121, 1945 (2007).

${ }^{13} \mathrm{~F}$. Yan and J. Rose, Aeronaut. J. 113(1144), 417-427 (2009).

${ }^{14} \mathrm{~W}$. Zhu and J. L. Rose, IEEE Trans. Ultrason. Ferroelectr. Freq. Control 46(3), 654-664 (1999).

${ }^{15}$ Z. Guo, J. Achenbach, and S. Krishnaswamy, Ultrasonics 35(6), 423-429 (1997).

${ }^{16}$ A. Safaeinili, O. Lobkis, and D. Chimenti, Ultrasonics 34(2), 393-396 (1996).

${ }^{17}$ C. Cosenza, S. Kenderian, B. B. Djordjevic, R. E. Green, and A. Pasta, IEEE Trans. Ultrason. Ferroelectr. Freq. Control 54(1), 147-156 (2007).

${ }^{18}$ R. A. Kruger, Med. Phys. 21(1), 127-132 (1994).

${ }^{19}$ F. L. Di Scalea, T. P. Berndt, J. B. Spicer, and B. B. Djordjevic, IEEE Trans. Ultrason. Ferroelectr. Freq. Control 46(6), 1551-1557 (1999).

${ }^{20}$ C. B. Scruby and L. E. Drain, Laser Ultrasonics Techniques and Applications (Taylor \& Francis, 1990).

${ }^{21}$ A. McKie, J. Wagner, J. Spicer, and C. Penney, Ultrasonics 27(6), 323-330 (1989).

${ }^{22}$ J. Huang, S. Krishnaswamy, and J. D. Achenbach, J. Acoust. Soc. Am. 92(5), 2527-2531 (1992).

${ }^{23}$ C. Edwards, A. Bushell, S. B. Palmer, and H. Nakano, Nondestr. Test. Eval. 10(1), 15-23 (1992).

${ }^{24}$ R. Pierce, C. Ume, and J. Jarzynski, Ultrasonics 33(2), 133-137 (1995).

${ }^{25}$ S. Pierce, B. Culshaw, and Q. Shan, Appl. Phys. Lett. 72(9), 1030-1032 (1998).

${ }^{26}$ J. Yang and C. Ume, Res. Nondestruct. Eval. 5(3), 175-190 (1994).

${ }^{27}$ T. Murray, J. Deaton, and J. Wagner, Ultrasonics 34 (1), 69-77 (1996).

${ }^{28}$ Y. H. Berthelot and J. Jarzynski, J. Nondestruct. Eval. 9(4), 271-277 (1990).

${ }^{29}$ E. F. Schubert and J. K. Kim, Science 308(5726), 1274-1278 (2005).

${ }^{30}$ T. J. Allen and P. C. Beard, Opt. Lett. 31(23), 3462-3464 (2006).

${ }^{31}$ R. G. M. Kolkman, W. Steenbergen, and T. G. van Leeuwen, Lasers Med. Sci. 21(3), 134-139 (2006).

${ }^{32} \mathrm{PCO}-7120$ data sheet, http://ixapps.ixys.com/DataSheet/pco-7120_data sheet.pdf (2003).

${ }^{33}$ I. A. Viktorov, Rayleigh and Lamb Waves: Physical Theory and Applications (1967).

${ }^{34}$ P. Moilanen, M. Talmant, V. Kilappa, P. Nicholson, S. Cheng, J. Timonen, and P. Laugier, J. Acoust. Soc. Am. 124, 2364 (2008). 